Related literature. The structure determination has established the stereochemistry of the compound (Aleksejczyk, Berchtold \& Braun, 1985) to be that displayed in Fig. 1 and represented by the structural formula:<smiles>OC1C=CC(O)C(Br)C1Br</smiles>

The Biomedical Research Support-Shared Instrumentation Grant Program, Division of Research
Resources, provided funds for the purchase of the diffractometer (NIH Grant S 10RR02243-01).

\section{References}

Aleksejczyk, R. A., Berchtold, G. A. \& Braun, A. G. (1985). J. Am. Chem. Soc. 107, 2554-2555.

ShELdRICK, G. M. (1978). Computing in Crystallography, edited by H. SCHENK, R. OlthOF-HAZEKAMP, H. van KonINGSVELD \& G. C. Bassi, pp. 34-42. Delft Univ. Press.

Silverman, L. D., Dewan, J. C., Giandomenico, C. M. \& LIPPARD, S. J. (1980). Inorg. Chem. 19, 3379-3383.

Acta Cryst. (1986). C42, 508-509

\title{
The Structure of the Norbornadiene* Adduct of 5-Phenyl-1,3,2,4,6-dithiatriazine
}

By A. W. Cordes, S. L. Craig and J. A. James Privett

Department of Chemistry, University of Arkansas, Fayetteville, AR 72701, USA

AND R. T. OAKLEY AND RENÉ T. BOERÉ

Guelph-Waterloo Centre for Graduate Work in Chemistry, Guelph Campus, Department of Chemistry and Biochemistry, University of Guelph, Guelph, Ontario, N1G 2W1 Canada

(Received 17 July 1985; accepted 29 October 1985)

Abstract. 10-Phenyl-1,8-dithia-9,11,12-triazatetracyclo[6.3.1.1 $\left.{ }^{3,6} \cdot 0^{2,7}\right]$ trideca-1(11),4,9,8(12)-tetraene, $\quad \mathrm{C}_{14^{-}}$ $\mathrm{H}_{13} \mathrm{~N}_{3} \mathrm{~S}_{2}, M_{r}=287.4$, monoclinic, $P 2_{1}, a=5.789$ (2), $b=10.064(1), c=11.109$ (2) $\AA, \beta=94.78(2)^{\circ}, V$ $=644.9$ (4) $\AA^{3}, Z=2, D_{x}=1.48 \mathrm{~g} \mathrm{~cm}^{-3}, \lambda($ Mo $K \alpha)$ $=0.71073 \AA, \quad \mu=3.84 \mathrm{~cm}^{-1}, \quad F(000)=300, \quad T=$ $293 \mathrm{~K}, R=0.037$ for 1203 unique observed reflections. The norbornadiene bonds to the sulfur atoms in the exo- $\beta$ orientation and the nitrogen atom between the sulfur atoms is displaced 0.802 (4) $\AA$ from the SNCNS plane. The $\mathrm{C}-\mathrm{N}$ bond lengths are equal $[1.335(5) \AA]$ and the $\mathrm{S}-\mathrm{N}$ bond lengths in a very narrow range of values $[1.637$ (4)-1.652 (4) $\AA]$.

Experimental. Compound prepared by the reaction of norbornadiene with 1,3-dichloro-5-phenyl-1,3,2,4,6dithiatriazine. Crystals obtained from acetonitrile solutions. White platelet data crystal $0.48 \times 0.04 \times$ $0.40 \mathrm{~mm}$ mounted on glass fiber. Intensities measured with Enraf-Nonius CAD-4 diffractometer, variablespeed $\omega-2 \theta$ scans. Unit cell from least squares of angle data for 18 reflections with $20<2 \theta<30^{\circ}$. Analytical absorption correction based on crystal shape varied from 0.89 to $1 \cdot 00$. Data collected to $\sin \theta / \lambda$ of

* IUPAC name: 8,9,10-trinorbornadiene.

0108-2701/86/040508-02\$01.50
$0.70 \AA^{-1},-8 \leq h \leq 8,0 \leq k \leq 14,0 \leq l \leq 15$. Three standard reflections $(060,224, \overline{2} 14)$ varied $\pm 1.9 \%$ over $18.6 \mathrm{~h}$ of data collection; anisotropic drift correction applied. 2061 reflections measured, 1972 unique $\left(R_{\text {int }}\right.$ $=0.03), 769$ reflections with $I<3 \sigma(I)$ considered unobserved. Solved by direct methods using MULTAN11/82 (Main, Fiske, Hull, Lessinger, Germain, Declercq \& Woolfson, 1982) and Fourier methods. Full-matrix least squares minimized $\sum w(\Delta F)^{2}$. $\mathrm{H}$ atoms refined with isotropic temperature factors, all other atoms refined anisotropically for 224 variables. $R=0.037, w R=0.043, S=1.09$, where non-Poisson $w^{-1}=\left[\sigma^{2}(I)+0.0025 I^{2}\right] / 4 F^{2}$. Final $(\Delta / \sigma)_{\max }<0.01$, $\Delta \rho_{\max }=0.21(5)$ and $\Delta \rho_{\min }=-0.24(5) \mathrm{e} \AA^{-3}$ on final difference map. Atomic scattering factors and anomalous-dispersion corrections from International Tables for X-ray Crystallography (1974) and programs used were those of Enraf-Nonius (1982) SDP.† Table 1 gives the atom coordinates and Table 2 selected bond distances and angles. Fig. 1 shows the molecule with the numbering scheme.

$\dagger$ Lists of structure factors and anisotropic thermal parameters have been deposited with the British Library Lending Division as Supplementary Publication No. SUP 42630 (23 pp.). Copies may be obtained through The Executive Secretary, International Union of Crystallography, 5 Abbey Square, Chester $\mathrm{CH} 12 \mathrm{HU}$, England.

(c) 1986 International Union of Crystallography 
Related literature. Structures of three other derivatives of 5-phenyl-1,3,2,4,6-dithiatriazine have recently been reported: $\mathrm{PhCS}_{2} \mathrm{~N}_{3} \mathrm{Cl}_{2}$ (Graham, Cordes, Oakley \& Boeré, 1985), $\mathrm{PhCS}_{3} \mathrm{~N}_{5}$ (Cordes, Oakley \& Boeré, 1985) and $\left(\mathrm{PhCS}_{2} \mathrm{~N}_{3}\right)_{2}$ (Boeré, French, Oakley, Cordes, Privett, Craig \& Graham, 1985). Other norbornadiene adducts of S-N rings include $\mathrm{Ph}_{2} \mathrm{~N}_{3} \mathrm{~S}_{2} \cdot \mathrm{C}_{7} \mathrm{H}_{8}$ (Burford, Chivers, Cordes, Laidlaw, Noble, Oakley \& Swepston, 1982), $\mathrm{Ph}_{3} \mathrm{NS}_{3} \mathrm{~N}_{3} \cdot \mathrm{C}_{7} \mathrm{H}_{8}$ (Liblong, Oakley, Cordes \& Noble,

Table 1. Fractional atomic coordinates, isotropic thermal parameters, and their e.s.d.'s

\begin{tabular}{|c|c|c|c|c|}
\hline & \multicolumn{4}{|c|}{$B_{\mathrm{eq}}=(4 / 3)\left(a^{2} B_{11}+b^{2} B_{22}+c^{2} B_{33}+a c B_{13} \cos \beta\right)$} \\
\hline & $x$ & $y$ & $z$ & $B_{\mathrm{eq}} / B\left(\AA^{2}\right)$ \\
\hline$S(1)$ & $0.1911(2)$ & 0.528 & $0.9143(1)$ & $3.92(2)$ \\
\hline$S(2)$ & $0.4552(2)$ & $0.3216(1)$ & $0.9175(1)$ & $3.59(2)$ \\
\hline$N(1)$ & $0.0401(6)$ & $0.4626(4)$ & 0.7969 (3) & $3.85(7)$ \\
\hline$N(2)$ & $0.2758(6)$ & $0.2629(4)$ & $0.8067(3)$ & $3.59(7)$ \\
\hline$N(3)$ & $0.4636(6)$ & $0.4841(4)$ & $0.9123(3)$ & $4 \cdot 32(8)$ \\
\hline$C(1)$ & $0.1032(7)$ & $0 \cdot 3416(4)$ & $0.7627(3)$ & $3 \cdot 18(7)$ \\
\hline$C(2)$ & $-0.0399(7)$ & $0.2871(4)$ & $0.6572(3)$ & $3.25(8)$ \\
\hline$C(3)$ & $0.0342(8)$ & $0.1776(5)$ & $0.5942(4)$ & $4 \cdot 2(1)$ \\
\hline$C(4)$ & -0.1024 (9) & $0.1244(6)$ & $0.4987(4)$ & $5 \cdot 2(1)$ \\
\hline$C(5)$ & $-0.315(\mathrm{l})$ & $0 \cdot 1800(6)$ & $0.4645(4)$ & $5.6(1)$ \\
\hline$C(6)$ & $-0.3883(8)$ & $0.2878(6)$ & $0.5260(5)$ & $5.4(1)$ \\
\hline$C(7)$ & $-0.2542(7)$ & $0.3418(5)$ & $0.6214(4)$ & $4.09(9)$ \\
\hline$C(8)$ & $0.1084(6)$ & $0.4220(4)$ & $1.0374(4)$ & $3.28(8)$ \\
\hline $\mathrm{C}(9)$ & $0.1561(9)$ & $0.4832(5)$ & $1 \cdot 1661(4)$ & $4.5(1)$ \\
\hline$C(10)$ & $0.0611(7)$ & $0.3781(6)$ & $1.2465(4)$ & $4.9(1)$ \\
\hline$C(11)$ & $0.2015(8)$ & $0.2787(5)$ & $1.2521(4)$ & $4.6(1)$ \\
\hline$C(12)$ & $0.3957(7)$ & $0.3100(5)$ & $1.1727(4)$ & $3.79(8)$ \\
\hline$C(13)$ & $0.2734(7)$ & $0.3009(4)$ & $1.0430(3)$ & $3.04(7)$ \\
\hline$C(14)$ & $0.4131(8)$ & $0.4602(5)$ & $1.1919(4)$ & $4.24(9)$ \\
\hline$H(3)$ & $0.157(6)$ & $0 \cdot 143(4)$ & $0.622(3)$ & $2.8(8)$ \\
\hline $\mathrm{H}(4)$ & $-0.058(7)$ & 0.049 (5) & $0.457(4)$ & $5(1)$ \\
\hline$H(5)$ & $-0.388(8)$ & $0.140(6)$ & $0.405(4)$ & $6(1)$ \\
\hline$H(6)$ & $-0.522(9)$ & $0.316(5)$ & $0.508(5)$ & $6(1)$ \\
\hline$H(7)$ & $-0.304(7)$ & $0.409(5)$ & $0.666(3)$ & $5(1)$ \\
\hline$H(8)$ & $-0 \cdot 066(6)$ & $0.396(4)$ & $1.008(3)$ & $2.9(8)$ \\
\hline$H(9)$ & $0.109(7)$ & $0.559(5)$ & $1.170(3)$ & $5(1)$ \\
\hline $\mathrm{H}(10)$ & $-0.094(8)$ & $0.388(6)$ & $1.272(4)$ & $7(1)$ \\
\hline $\mathrm{H}(11)$ & $0.180(7)$ & $0.192(5)$ & $1.276(4)$ & $6(1)$ \\
\hline $\mathrm{H}(12)$ & $0.514(7)$ & $0.255(5)$ & $1.179(4)$ & $5(1)$ \\
\hline$H(13)$ & $0.191(7)$ & $0.221(4)$ & $1.023(3)$ & $4(1)$ \\
\hline$H(14 A)$ & $0.466(7)$ & $0.475(5)$ & $1 \cdot 270(4)$ & $5(1)$ \\
\hline$H(14 B)$ & $0.508(6)$ & $0.502(4)$ & $1.146(3)$ & $3.7(9)$ \\
\hline
\end{tabular}

Table 2. Selected bond distances $(\AA)$ and bond angles $\left(^{\circ}\right)$ and their e.s.d.'s

$\begin{array}{lll}S(1) & C(8) & 1.831(5) \\ S(1) & N(1) & 1.647(4) \\ S(1) & N(3) & 1.641(4) \\ S(2) & C(13) & 1.827(4) \\ S(2) & N(2) & 1.652(4) \\ S(2) & N(3) & 1.637(4) \\ N(1) & C(1) & 1.336(5) \\ N(2) & C(1) & 1.335(5) \\ C(1) & C(2) & 1.483(6)\end{array}$

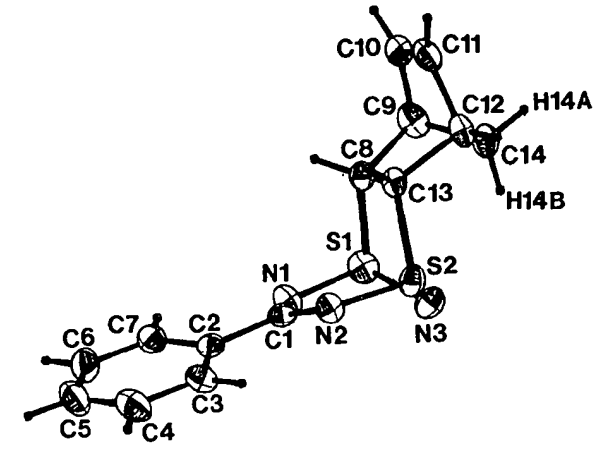

Fig. 1. ORTEPII diagram (Johnson, 1976) and atom-numbering scheme. Non- $\mathrm{H}$ ellipsoids at $30 \%$ probability level, $\mathrm{H}$ atoms given arbitrary radii.

$1983)$ and $\left(\mathrm{CF}_{3}\right)_{2} \mathrm{PS}_{2} \mathrm{~N}_{3} \cdot \mathrm{C}_{7} \mathrm{H}_{8}$ (Roesky, Lucas, Noltemeyer \& Sheldrick, 1984).

We thank the National Science Foundation, the State of Arkansas, the Research Corporation and the Natural Sciences and Engineering Research Council of Canada for financial support.

\section{References}

Boeré, R. T., French, C. L., Oakley, R. T., Cordes, A. W., Privet, J. A. J., Craig, S. L. \& Graham, J. B. (1985). J. Am. Chem. Soc. 107, 7710-7717.

Burford, N., Chivers, T., Cordes, A. W., LaIDlaw, W. G., Noble, M. C., OAKLeY, R. T. \& Swepston, P. N. (1982). J. Am. Chem. Soc. 104, 1282-1290.

Cordes, A. W., OAKley, R. T. \& Boeré, R. T. (1985). Acta Cryst. C41, 1833-1834.

Enraf-Nonius (1982). Structure Determination Package. EnrafNonius, Delft.

Graham, J. B. III, Cordes, A. W., OAkley, R. T. \& Boeré R. T. (1985). Acta Cryst. C41, 1835-1836.

International Tables for X-ray Crystallography (1974). Vol. IV. Birmingham: Kynoch Press. (Present distributor D. Reidel, Dordrecht.)

JOHNSON, C. K. (1976). ORTEPII. Report ORNL-5138. Oak Ridge National Laboratory, Tennessee.

Liblong, S. W., OAKley, R. T., Cordes, A. W. \& Noble, M. C. (1983). Can.J. Chem. 61, 2062-2067.

Main, P., Fiske, S. J., Hull, S. E., Lessinger, L., Germain, G., DEClERCQ, J.-P. \& WOOLFSON, M. M. (1982). MULTAN11/82. A System of Computer Programs for the Automatic Solution of Crystal Structures from $X$-ray Diffraction Data. Univs. of York, England, and Louvain, Belgium.

Roesky, H. W., Lucas, J., Noltemeyer, M. \& Sheldrick, G. M. (1984). Chem. Ber. 117, 1583-1590. 